\title{
Expanded endonasal approach: the rostrocaudal axis. Part I. Crista galli to the sella turcica
}

\author{
Amin Kassam, M.D., Carl H. Snyderman, M.D., Arlan Mintz, M.Sc., M.D., \\ Paul Gardner, M.D., and Ricardo L. Carrau, M.D. \\ Departments of Neurosurgery and Otolaryngology, Minimally Invasive Neurosurgery Center, and \\ Center for Cranial Base Surgery, University of Pittsburgh Medical Center, Pittsburgh, Pennsylvania
}

\begin{abstract}
Object. Transsphenoidal approaches have been used for a century for the resection of pituitary and other sellar tumors. More recently, the standard endonasal approach has been expanded to provide access to other, parasellar lesions. With the addition of the endoscope, this expansion carries significant potential for the resection of skull base lesions.

Methods. The anatomical landmarks and surgical techniques used in expanded (extended) endoscopic approaches to the rostral, anterior skull base are reviewed and presented, accompanied by case illustrations of each segment (or module) of approach. The rostral half of the anterior skull base is divided into modules of approach: sellar/parasellar, transplanum/transtuberculum, and transcribriform. Case illustrations of successful resections of lesions with each module are presented and discussed.

Conclusions. Endoscopic, expanded endonasal approaches to rostral anterior skull base lesions are feasible and hold great potential for decreased morbidity. The effectiveness and appropriate use of these techniques must be evaluated by close examination of outcomes as case series expand.
\end{abstract}

\section{KEY WORDS • endoscopic surgery • skull base • endonasal approach • transsphenoidal approach}

Transsphenoidal approaches to the ventral midline skull base were first proposed more than a century ago. Initially these approaches were restricted to the pituitary fossa, $, 5,10,14$ but with progressive evolution in biotechnology $y^{7,8}$ coupled with increased anatomical understanding, the transsphenoidal approaches were extended to regions beyond the sella turcica $a^{4,6,15}$ to include other entities in addition to pituitary tumors. The introduction of the operating microscope by Hardy in the 1960s, ${ }^{9}$ coupled with Dott's contribution of fluoroscopy, ${ }^{14}$ provided the first critical navigation/visualization integration that formed the foundation of the work that followed.

The addition of the endoscope, ${ }^{1-3,7,11-13}$ coupled with the current sophisticated neuronavigation systems, builds on this foundation. The expanded endonasal approach evolved when these principles were then further augmented with the concept of team surgery; that is, a neurosurgeon and otolaryngologist working simultaneously throughout all phases of the surgery (approach, resection, and reconstruc-

Abbreviations used in this paper: $\mathrm{ACA}=$ anterior cerebral artery; $\mathrm{ACoA}=$ anterior communicating artery; $\mathrm{AEA}=$ anterior ethmoidal artery; $\mathrm{CA}=$ carotid artery; $\mathrm{CSF}=$ cerebrospinal fluid; $\mathrm{ICA}=$ internal carotid artery; $\mathrm{MR}=$ magnetic resonance; $\mathrm{OCR}=$ opticocarotid recess; $\mathrm{PEA}=$ posterior ethmoidal artery; $\mathrm{SIS}=$ superior intercavernous sinus. tion). Using the principles of the expanded endonasal approach, we are now able to access the entire ventral skull base, from the crista galli up to and through the odontoid, and we are able to address a diverse array of intra- and extradural entities.

In this report we describe our experience over the past 7 years, accessing the ventral skull base along a midline sagittal plane by using a fully endoscopic, completely transnasal approach. Our system of modular approaches is based on specific anatomical corridors. For each module we will outline the key anatomical principles and technical nuances, using illustrative case examples to highlight these principles.

\section{GENERAL APPROACH}

\section{Patient Positioning}

Patients are positioned supine on the operating table in Mayfield head holders, with the head in a neutral position and slightly rotated to the right side. The patient position remains the same for each approach. For more rostral or caudal lesions, $45^{\circ}$ or $70^{\circ}$ endoscopes may be used. The monitors used for image guidance and endoscopy are positioned directly in front of the surgeons. 
The nose is prepared by placing pledgets soaked with $0.02 \%$ oxymetazoline into each naris, followed by povidone iodine solution applied over the nose and upper lip as well as into each nares on a cotton tip applicator.

\section{Bilateral Exposure}

Bimanual dissection forms the foundation of microneurosurgery and is also an absolute prerequisite for endoneurosurgery. To allow for bimanual dissection, a binasal approach is strongly advocated for all expanded endoscopic approaches. The procedure is initiated in the right naris with the removal of the middle turbinate. For routine transsellar exposures for microadenomas, the middle turbinate may be preserved. The posterior septum is disarticulated from the rostrum of the sphenoid bone, which is then removed to create a bilateral opening in the sphenoid sinus. The medial turbinate of the left naris is generally lateralized but not resected. The natural sphenoid ostium on the left is opened and widened so that there is communication with the previous sphenoidotomy on the right side, thus creating wide bilateral sphenoidotomies. The lateral margins of the sphenoidotomies are extended to the level of the medial pterygoid plates.

The endoscope is then returned to the right naris and a small portion $(\sim 1-2 \mathrm{~cm})$ of the posterior nasal septum is resected. This represents the most critical step in the binasal approach because it facilitates bilateral instrumentation without deviation of the septum into the path of the endoscope and compromise of visualization (Video 1). The bilateral sphenoidotomies made via the binasal approach form the foundation of all subsequent expanded endoscopic approach modules. To optimize the available space, the endoscope is positioned superiorly at 12 o'clock, with the suction entering into the 6-o'clock position in the right naris (Fig. 1). Dissecting instruments can be introduced into the nasal cavity through the left naris (Video 1).

Video 1. Principles of general bilateral exposure. (Click here to view with Windows Media Player and a broadband

connection here to view with a dial-up connection, or here to

view with RealPlayer.)

The principle of this exposure is to create a single large rectangular cavity within the sphenoid sinus. This allows for the progressive placement of the endoscope closer to the target, which is critical for visualization by allowing for the delivery of divergent light ("flash-light" effect), close to the target and its magnification. Close placement of the endoscope is also imperative in the event of bleeding to allow for maneuverability of the equipment without soiling its lens, which can be a frustrating limitation when working through a tight corridor.

\section{Resection Procedures}

Intradural Endoneurosurgical Dissection Techniques. Endoneurosurgical tumor resection uses microneurosurgical techniques identical to those of internal debulking, capsular mobilization, and extracapsular dissection of neurovascular structures, along with coagulation and capsule removal. These principles have stood the test of time, minimize morbidity, and are primary approaches for the removal of any tumor. It must be emphasized that these endoneurosurgical dissection techniques are of critical importance and take precedence over the degree of tumor resection in all circumstances.

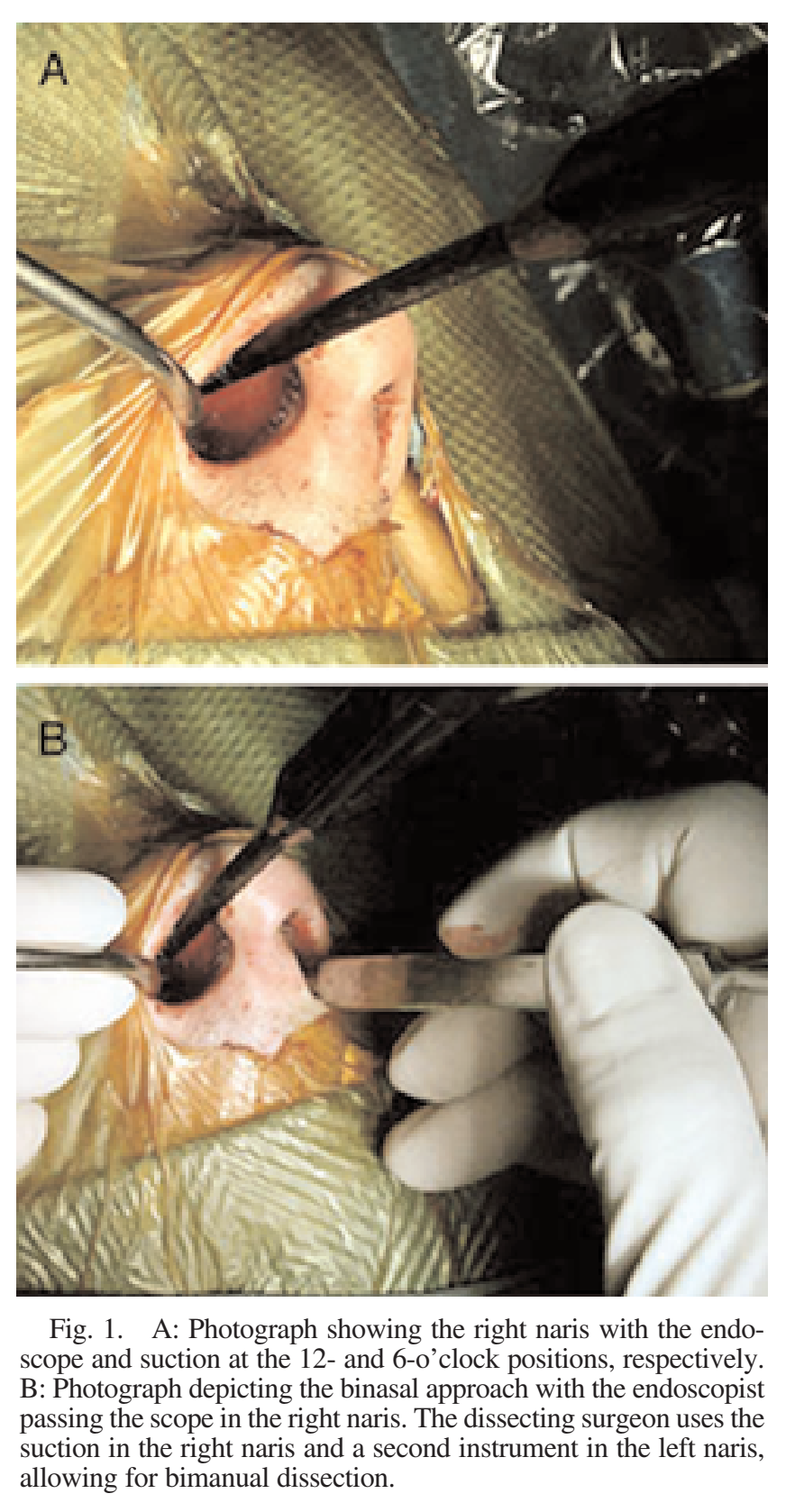

Tumor debulking techniques are dependent on tumor consistency. When feasible, a technique in which two suction devices are used has proven to be the safest means for debulking. For a right-handed surgeon, a suction device is used in the left hand to maintain gentle countertraction while a dissecting or debulking instrument is used in the right. Countertraction exerted with a grasping instrument can tear extracapsular vessels that will retract and bleed. For debulking of soft lesions, such as pituitary tumors, a No. 6 or at most a No. 8 French suction is adequate. Malleable suctions allow for distal angulation to improve access. For firmer tumors, an ultrasonic aspirator can be used. Rarely, the tumor contents can be removed piecemeal intracapsularly by using fine cup forceps or pituitary forceps. Internal debulking is continued until the capsule is mobile. 
Extracapsular dissection should be done sharply, or alternatively, with fine blunt endoscopic dissectors or small suction device (Video 2). Critical neurovascular structures are then identified and protected. The capsule is coagulated using the appropriately shaped endoscopic bipolar cautery. Care should be taken to avoid thermal injury to important neurovascular structures from heat dispersal during cauterization.

Video 2. Principles of endoneurosurgery. (Click here to view with Windows Media Player and a broadband connection, here to view with a dial-up connection, or here to view with RealPlayer.)

\section{SPECIFIC MODULES}

\section{Pituitary and Sellar Surgery}

Illustrative Case. This 64-year-old woman presented with progressive visual loss. Neuroendocrine function was found to be intact. Admission MR imaging revealed a large pituitary macroadenoma (Fig. 2). The patient underwent an endoscopic tumor removal with preservation of the pituitary gland and recovery of visual function. This case is presented to demonstrate the specific exposure required and the sequence for the intrasellar dissection technique.

\section{Technical Considerations.}

Exposure. Once the general exposure is achieved (see General Approach, subhead Bilateral Exposure) and bilateral sphenoidotomies are completed, the exposure of the pituitary fossa can proceed. The sphenoidotomy is widened to include the lateral recess of the sphenoid extending lateral to the carotid canal. The exposure is then extended ros-
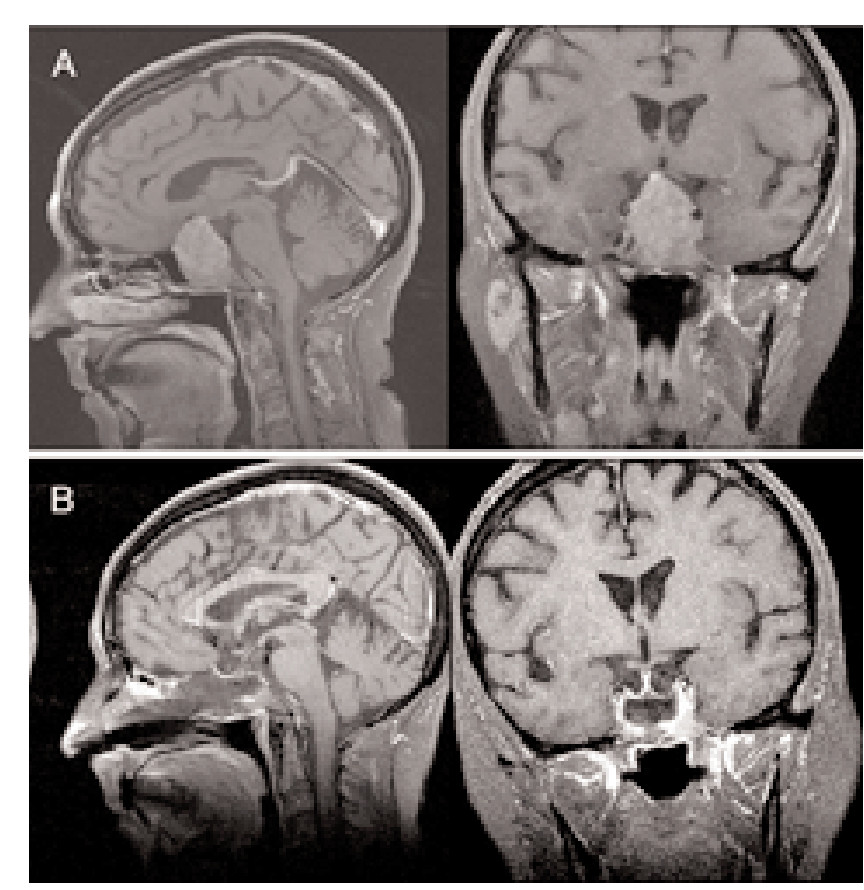

Fig. 2. A: Preoperative contrast-enhanced sagittal and coronal MR images. B: Postoperative contrast-enhanced sagittal and coronal MR images. Notice the residual gland on the coronal image located at the level of the right OCR. trally to expose the posterior cells of the ethmoid sinus and to further define the planum-tuberculum junction. Finally, the floor of the sphenoid is reduced back to the level of the clivus. This is particularly important in the case of macroadenomas with significant suprasellar extension. By reducing the floor of the sphenoid, a greater caudal-to-rostral trajectory into the suprasellar space is created. Any intrasphenoidal septations must then be reduced with care, because the paramedian septations often lead to the vertical canal of the ICA. The sphenoid sinus mucosa is removed and the venous bleeding is controlled by irrigation with warm saline.

This approach generates the desired single rectangular cavity and allows for the identification of key anatomical landmarks (Fig. 3) as follows: the medial OCR, the carotid protuberance within the parasellar space, the sellar face, clival recess, and the strut of bone over the SIS. The medial OCR represents the ventral surface of the medial clinoid and is the key anatomical landmark in this region. An entry at the level of the medial OCR will allow for simultaneous access to the carotid canal, optic canal, sella turcica, and medial cavernous sinus. It is analogous to a "key hole" during a conventional pterional craniotomy.

The medial OCR does not need to be opened for the removal of pituitary tumors unless there is significant suprasellar and lateral extension toward the opticocarotid cistern. Removal of the bone overlying the SIS is imperative for lesions that extend into the anterior cranial fossa. Therefore, bone removal over the sellar face should extend laterally over the medial portions of each cavernous sinus and rostrocaudally to expose both the superior and inferior intercavernous sinus. It has been our experience that surgeons

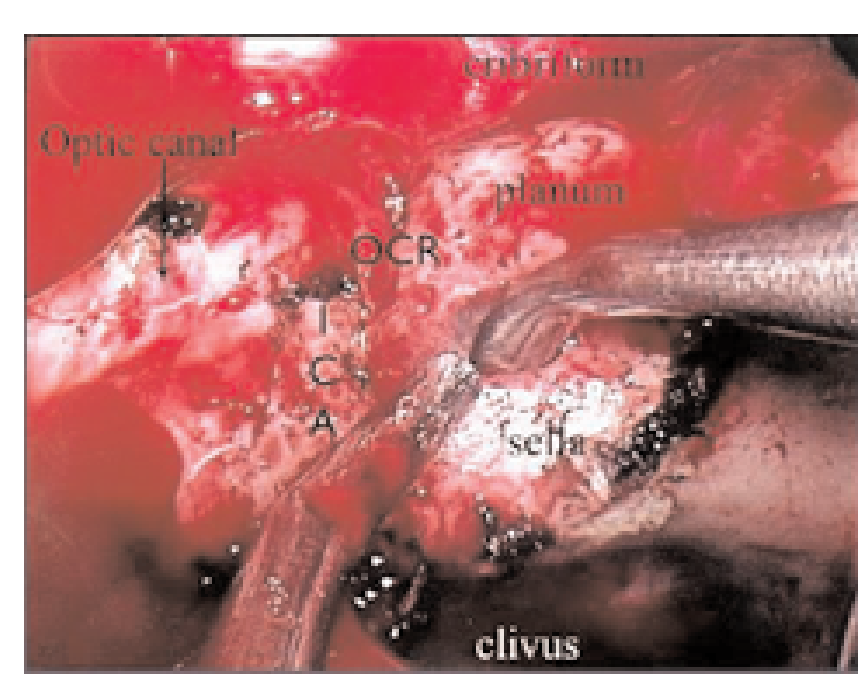

Fig. 3. Endoscopic view demonstrating the expanded endonasal approach used to access the anterior skull base. The initial module exposes the sellar floor rostrally to the level of the SIS (dissector). The protuberance created by the parasellar ICA extends vertically to meet the optic canal that is obliquely traveling from medial to lateral in a posterior to anterior direction. Medial to this junction is the medial OCR, which represents the key anatomical landmark for all anterior base modules. This sellar module can be progressively extended in a rostral direction by performing wide bilateral ethmoidectomies to expose the planum sphenoidale and the cribriform plate sequentially. 
who are novices at endoscopy fail to undertake an adequate exposure and are content with a smaller window. This eliminates the primary advantage of endoscopic surgery, which is to deliver divergent focal light and magnification that facilitates superior visualization and dissection.

Intrasellar Dissection. We prefer not to use curettes for tumor removal but instead to rely on the endoneurosurgical techniques described earlier. Although extracapsular dissection for pituitary tumors is often not possible, debulking using two suctions or an expanded endonasal ultrasonic aspirator in our opinion provides for a more controlled removal with improved and less traumatic separation of the tumor from the normal gland, stalk, and cavernous sinus contents. It should be noted that the improved visualization provided by the endoscope does allow for extracapsular dissection in many cases.

As macroadenomas are resected, the diaphragma will descend and obstruct visualization. Therefore, systematic intrasellar dissection is mandatory to optimize visualization while protecting the normal gland. The dura mater is opened using a sickle knife in the center of the sellar face. The opening is extended caudally and obliquely toward the 8and 5-o'clock positions, creating the inferior flap of the opening. The superior flap is left intact to act as a retractor and hold the anterior face of the tumor overlying the diaphragma (Fig. 4A). In the case of a macroadenoma, the tumor will herniate through this inferior opening. This portion of the tumor is removed in a posterior trajectory toward the clivus-dorsum junction. The resection should extend widely from cavernous sinus to cavernous sinus laterally and posteriorly to the dorsum. Care should be taken because the bone is defective posteriorly in many macroadenomas, and the basilar cistern can be transgressed. The pituitary stalk is most commonly located in this position.

Once the posterolateral dissection is completed, the superior dura mater can now be opened, allowing access to the superior portion of the tumor (Fig. 4B). The superolateral dissection along the cavernous-carotid recess can then begin. Each recess is dissected from a caudal-to-rostral direction vertically. Before proceeding superiorly, the posterior junction of the cavernous-carotid recess should be examined and the tumor posterior to the carotid genu in the cavernous sinus can be removed (see Cavernous Sinus Extension). Often venous bleeding is encountered during this

Fig. 4. Endoscopic views demonstrating the critical segments of intrasellar tumor removal. A: The superior dural leaf is left intact to allow for the tumor (T) to herniate through the initial inferior (I) opening. The inferior portion of the tumor is then resected in a posterior direction toward the clival dorsal junction. B: The superior leaf of the dura mater (DM) is opened with scissors (S) after the inferior portion of the tumor has been removed. C: Endoscopic view of the superior right margin. The diaphragma (D) is retracted medially and the right optic nerve $(\mathrm{O})$ can be seen through the diaphragma. The ICA in the cavernous sinus can be seen traveling laterally to form the OCR. D: Following removal of the anterior and superior portions of the tumor the diaphragma will descend. This is then gently retracted superiorly and the endoscope can be brought directly into the cavity to examine the lateral margins. In this view the left ICA can be seen because the medial cavernous sinus wall has been eroded. Tumor in the posterior $(\mathrm{P})$ segment of the cavernous sinus behind the ICA can then be removed.
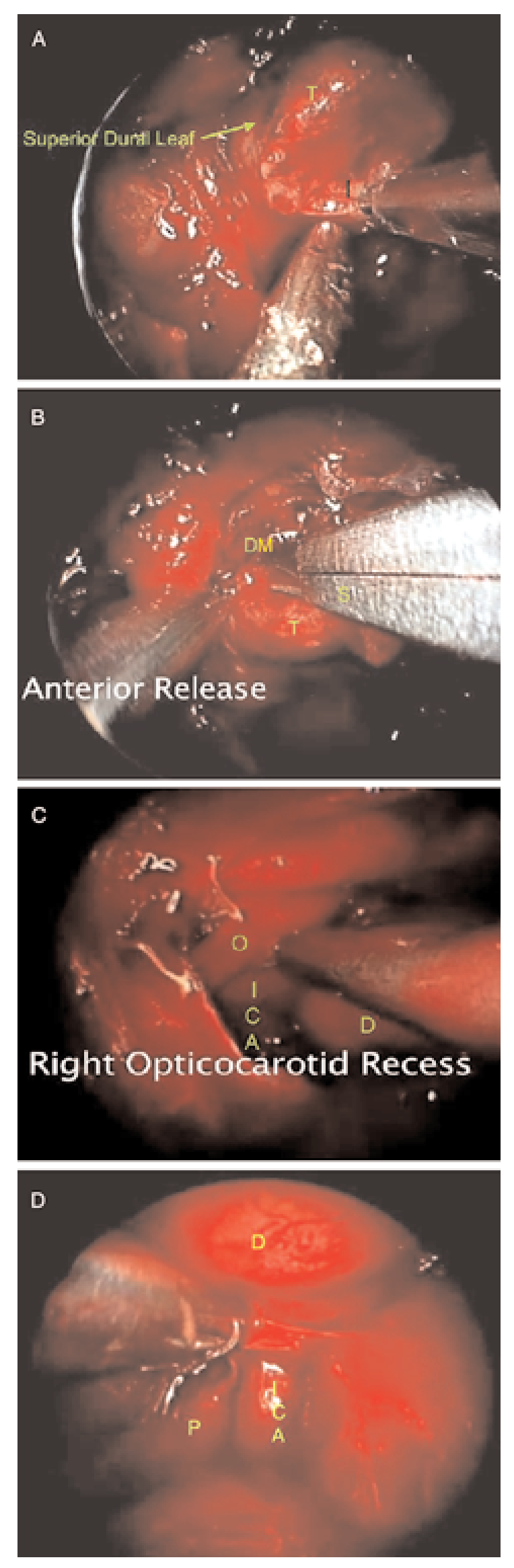
stage, and can be managed with packing. This should be done prior to dissecting superiorly and risking a CSF leak to avoid the exchange of blood for CSF. Once the superior lateral portion of the tumor in the sella turcica is removed, the medial OCR and angle between the optic nerve and CA as seen from inside the sella superolaterally should be examined carefully bilaterally (Fig. 4C). This is one of the two sites most commonly found for retained tumor. The other is under the anterior lip of the dura mater at the level of the SIS directly beneath the sellar/tuberculum junction. To optimize visualization of this segment, the tumor along the anterior face overlying the diaphragma can be removed. This will allow the diaphragma to descend, opening up the superior angle between the dura mater at the level of the SIS and the anterior attachment of the diaphragma. The dura can be coagulated, shrinking it back to the SIS. These maneuvers in combination with removal of the overlying bone will optimize visualization.

Final inspection of the sella turcica is undertaken in a clockwise fashion, starting inferiorly at 6 o'clock and using angled endoscopes as required. At this stage, the diaphragma should descend concentrically. In our experience, the most common position of the residual gland is as an apron plastered to the undersurface of the diaphragma. If the diaphragma fails to descend concentrically, this is indicative of retained tumor in the suprasellar space. Depending on the goals of surgery, however, this may be an acceptable outcome. If the goals are optic decompression or staged surgery, then tumor removal should not be considered adequate until pulsations are visible in the diaphragma; in our experience this is a robust finding. If descent is not concentric and additional removal is needed, the diaphragma is electively opened. With the aid of the endoscope, residual tumor in the suprasellar cistern can be removed until the optic nerves are directly visualized. During this resection, we strongly urge the use of endosurgical dissection techniques previously described as opposed to curettes, because small subchiasmatic and infundibular perforating vessels must be preserved. If such suprasellar dissection is required then it is imperative to remove the bone overlying the medial OCR completely. This allows identification of the optic nerve and CA laterally and facilitates extracapsular dissection without the need for excessive countertraction. These maneuvers will maximize the chance of preserving the perforating vessels (Video 3).

Video 3. Intrasellar resection technique. (Click here to view with Windows Media Player and a broadband connection here to view with a dial-up connection, or here to view with

RealPlayer.)

Cavernous Sinus Extension. Once the superior and inferior margins of the cavity have been examined, the endoscope can be place directly within the sella to examine the medial cavernous walls that form the lateral margins of the cavity. Visualization is improved in macroadenomas by the sellar expansion but can be augmented with angled endoscopes if needed. The improved visualization facilitates the elective opening of the cavernous sinus to access tumor extension within the medial segment. The carotid siphon is usually anteriorly placed and the space between the posterior clinoid and the siphon represents an ideal entry point (Fig. 4D). Most often, the tumor creates a pathway through this space that can be pursued. The tumor posterior to the
CA can be removed using the two-suction technique. As a note of caution, if there is an opening in the diaphragma (intentional or inadvertent), great care should be taken to avoid the exchange of blood for CSF and to avoid a subarachnoid hemorrhage with its associated complications.

\section{EXPANDED APPROACHES}

Based on our pituitary experience we then proceeded to apply these principles to create a series of modular expanded approaches. The natural extension was rostrally to access the suprasellar cistern and anterior cranial base directly without going through the sella turcica. The transtuberculum/transplanum and transcribriform approaches are described next.

\section{Transtuberculum/Transplanum Approach}

Illustrative Case. This 40-year-old woman presented with progressive visual failure (Fig. 5A). Admission MR imaging revealed a suprasellar meningioma with significant optic chiasm compression (Fig. 6A). Complete tumor resection was achieved via an expanded endonasal approach transplanum module (Fig. 6B). The patient was observed postoperatively for 72 hours and discharged. On follow-up examination, visual fields demonstrated a complete recovery (Fig. 5B). This case is presented to demonstrate the exposure required and the nuances of the intradural dissection technique.

\section{Technical Considerations.}

Extradural Exposure. Once the general bilateral approach to the sphenoid is completed, the rostral exposure is initiated by completing wide bilateral anterior and posterior ethmoidectomies (Fig. 3). The ethmoid septations are drilled flush with the anterior cranial base and laterally to the level of the lamina papyracea, providing for a wide lateral exposure. For a transplanum approach, we try to minimize the risk of compromising olfaction by ensuring that the anterior margin of the exposure does not extend rostrally to the level of the PEAs and canals, and we leave the most rostral margin of the nasal septum attached to the skull base.

Next, the planum sphenoidale is drilled from a rostral-tocaudal direction. Once the planum is eggshell thin it can be gently fractured inferiorly by using a blunt dissector or Kerrison rongeurs, if needed. The most rostral portion of the sellar floor can be opened to expose the SIS. To mobilize the SIS, the overlying bone strut is made eggshell thin by the drill and fractured inferiorly (alternatively, a 1-mm, $45^{\circ}$ Kerrison rongeur can be used). Mobilization of the sinus will allow for subsequent direct access to the suprasellar portion of the tumor in the parachiasmatic cisterns. Removal of this segment of the bone is adequate to allow for direct access without the need to transect the SIS. Sinus bleeding can be controlled with microfibrillar collagen "sandwiches." The ventral aspect of the medial clinoids, representing the medial OCR, is drilled away with a 3-mm hybrid bit, with copious irrigation to protect the optic nerves from thermal injury. It is not uncommon to find venous bleeding from the SIS as it inserts laterally into the cavernous sinus directly under this bone. Using a 1-mm Kerrison rongeur, the paraclinoid carotid canals can be removed in this region. This should be done by using only 

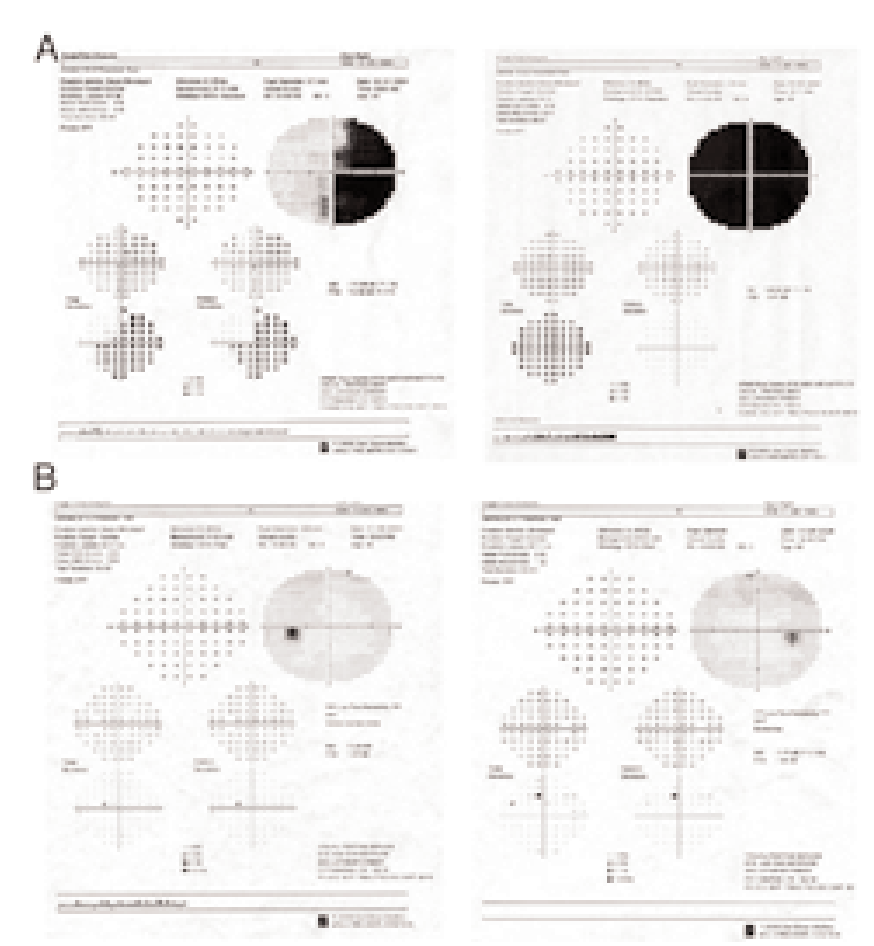

Fig. 5. A: Preoperative visual field charts showing severe field deficits. B: Postoperative visual field charts showing visual recovery. one third of the distal tip of the Kerrison positioned vertically in a parallel orientation to the underlying subclinoid carotid canal. Removing the remainder of the medial clinoid process is imperative to be able subsequently to access the OCR intradurally without having to retract the tumor and risk injury to the perforating artery. This point cannot be overemphasized (Video 4).

Video 4. Planum exposure. (Click here to view with Windows Media Player and a broadband connection here to view with a dial-up connection, on here to view with RealPlayer.)

Tumor Devascularization. It is not uncommon in anterior skull base meningiomas to find a feeding artery arising from the distal portion of the paraclinoid CA at the level of the medial OCR. This feeding vessel is coagulated to aid in the devascularization of the tumor. Exposing and sacrificing the PEAs further accomplishes this goal. These arteries are located approximately 5 to $7 \mathrm{~mm}$ anterior to the orbital apex. The posterior ethmoidal canal is identified as it emerges from the lamina papyracea and courses toward the fovea ethmoidalis. The canal is unroofed to identify the PEA, which is then ligated. Care should be taken to prevent the proximal stump from bleeding and retracting into the orbit, resulting in a retrobulbar hematoma. The PEA is ligated using the technique described in the Transcribriform module to secure the AEA. At this point, a single midline anterior base cavity has been created that extends from the
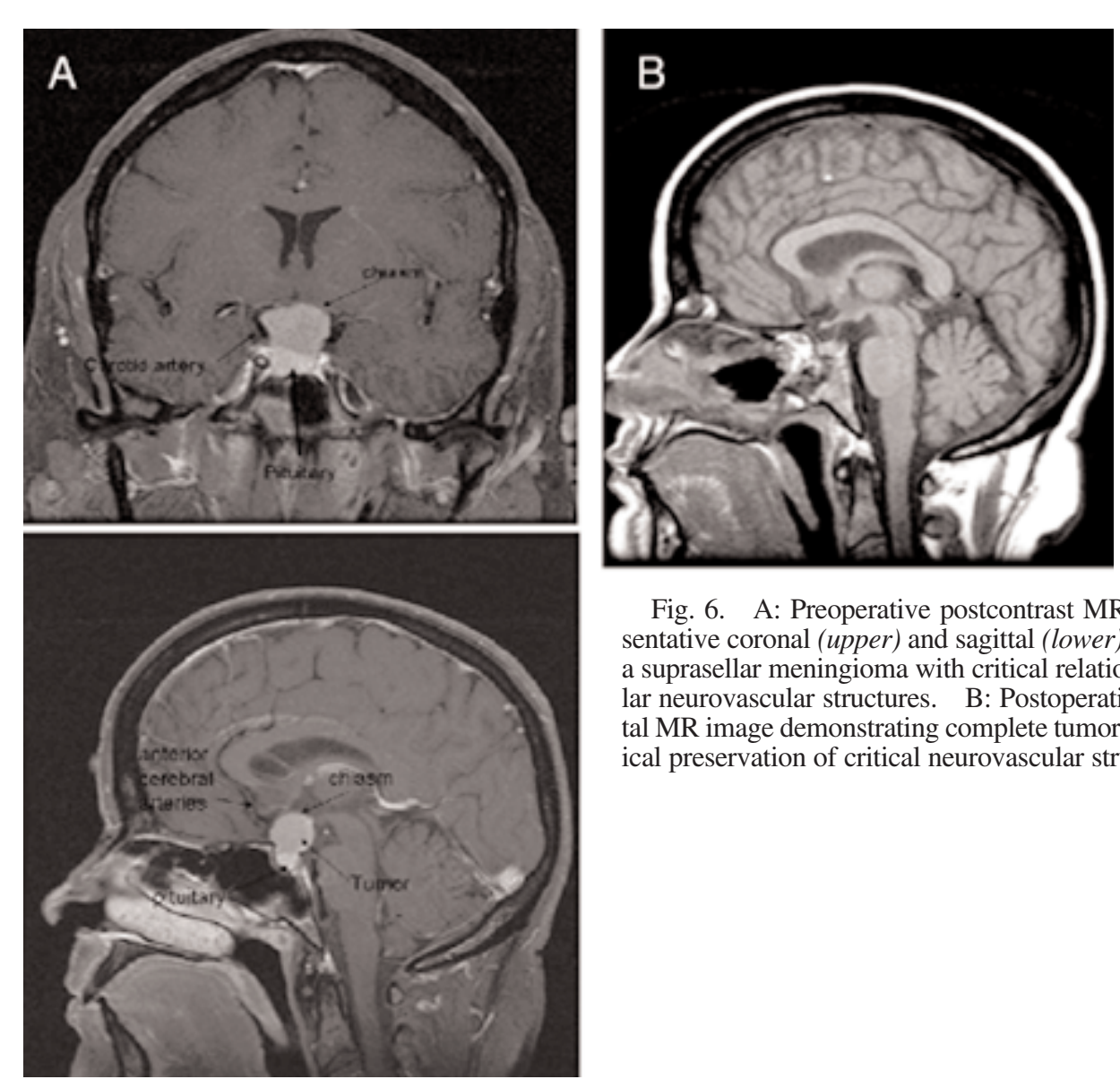

Fig. 6. A: Preoperative postcontrast MR imaging with representative coronal (upper) and sagittal (lower) views demonstrating a suprasellar meningioma with critical relationships to the parasellar neurovascular structures. B: Postoperative postcontrast sagittal MR image demonstrating complete tumor removal and anatomical preservation of critical neurovascular structures. 
planum/cribriform junction to the clival recess anteroposteriorly and laterally between each lamina papyracea. The exposed dura mater extending from the sella turcica to the anterior portion of the planum is then meticulously and tediously coagulated to provide further devascularization (Fig. 7A). The dura mater is opened widely in a cruciate fashion, taking care not to transgress the prechiasmatic cisterns (Fig. 7B). Excessive exposure will allow normal brain to herniate through the defect and obscure visualization. The anterior margin of the lesion is identified and the brain/tumor interface marks the extent of the opening required. Additional bone and dura mater that interferes with the exposure of this junction it should be removed at this stage.

Intradural Dissection. Using the principles of endoneurosurgical dissection, systematic extracapsular dissection through the parachiasmatic cisterns is accomplished. Critical neurovascular structures are identified. The first landmark sought should be the paraclinoid CA as it emerges intradurally at the level of medial OCR, over which the bone should have already been adequately removed (Fig. 8). The CA will lead to the optic nerve, which is located slightly superiorly. The optic nerve in turn can be followed with circumferential extracapsular dissection to the chiasm and then to the contralateral optic nerve and CA. It bears repeating that to undertake extracapsular dissection safely in this region, the capsule of the tumor must be thinned enough to allow for gentle traction with no more than a No. 4 or 6 French catheter used for suction. This will allow the arachnoid bands of the parachiasmatic cisterns to be under enough tension to facilitate sharp dissection (Fig. 9). Finally, care should be taken during the parachiasmatic dissection, because the ACoA and in particular the recurrent artery of Huebner may be draped over the superior surface.

Once the suprachiasmatic dissection has been completed the subchiasmatic sequence can begin. During this sequence, several additional critical steps need to be specifically performed to preserve critical structures during the extracapsular dissection. These are performed once adequate debulking in the respective regions has been accomplished. Small subchiasmatic perforating vessels are often draped around the circumference of the tumor (Fig. 10). With adequate debulking and mobilization these can usually be spared, and if present, the branches directly feeding the tumor can be coagulated and sectioned (Fig. 11).

The infundibulum is most often adherent to the posterior margin of the capsule and can easily be damaged during coagulation of the base of the tumor at the tuberculum/sellar junction. Bipolar cauterization should be avoided in this space until the stalk has been specifically identified (Fig. 12, Video 5).

Video 5. Intradural transplanum dissection. (Click here to view with Windows Media Player and a broadband connection, here to view with a dial-up connection, or here to view with RealPlayer.)

\section{Transcribriform Approach}

Illustrative Case. This 64-year-old woman presented with visual failure, anosmia, and severe neurocognitive deficits. Preoperative MR imaging revealed a large olfactory groove meningioma with severe bifrontal edema (Fig. 13A). Com- puterized tomography angiography scans demonstrated significant vascular adherence of the second segments of the ACAs $\left(\mathrm{A}_{2}\right)$ to the capsule of the tumor (Fig. 13B). The patient underwent a staged expanded endoscopic transcribriform surgery for tumor removal via a fully endoscopic transnasal approach Fig. (13C). Postoperatively the patient made a complete visual recovery and her neurocognitive deficits resolved completely. This case is presented to
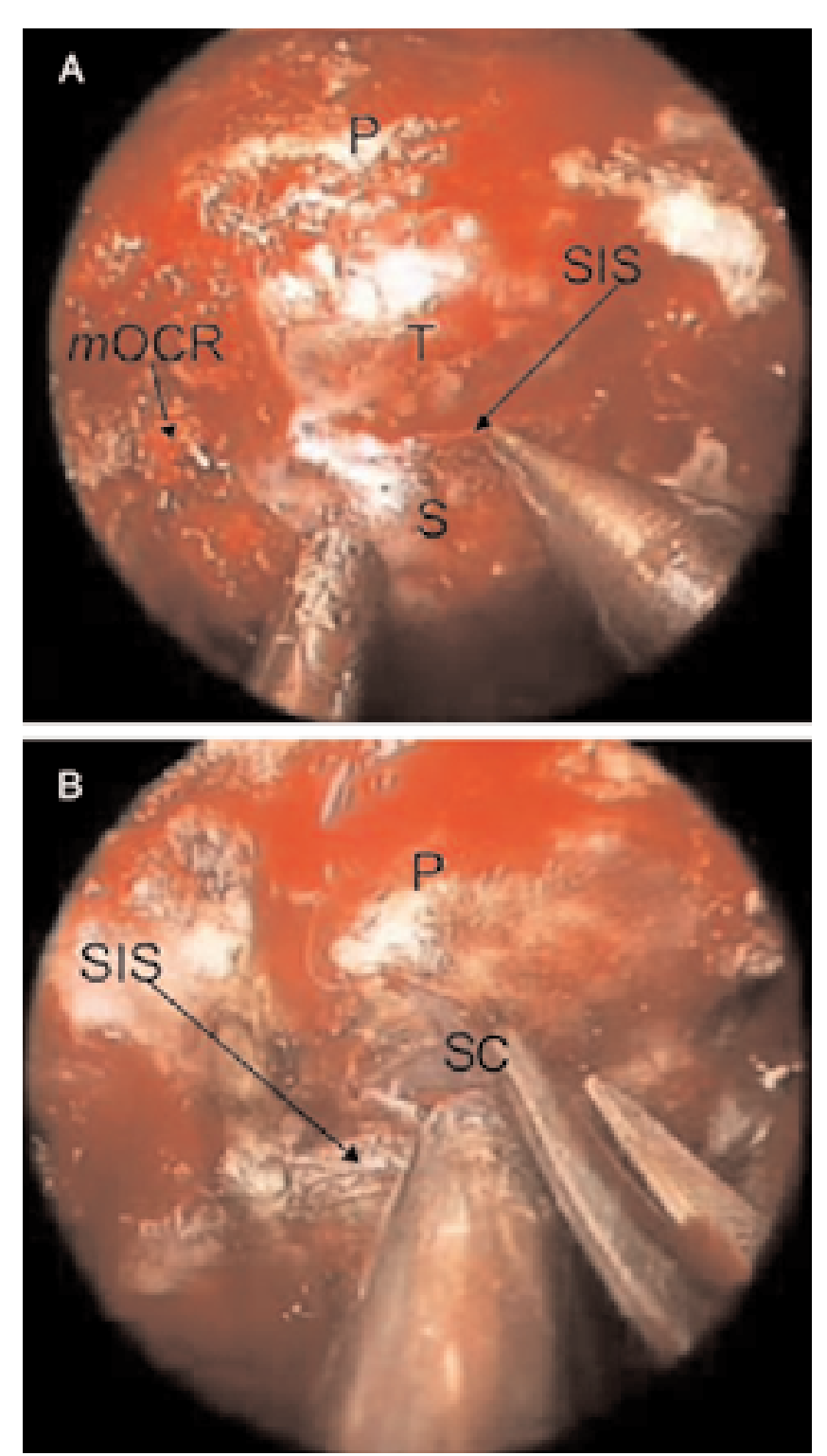

Fig. 7. A: Endoscopic view of the planum module once the bone removal has been completed. In the rostrocaudal axis the planum sphenoidale $(\mathrm{P})$, tuberculum $(\mathrm{T})$, and sella turcica $(\mathrm{S})$ can be seen. The fold of the SIS between the tuberculum and sella is seen as well. The lateral extent of the opening is to the junction of the SIS as it merges with the cavernous sinus. This represents the region of the medial OCR (mOCR). B: Endoscopic view showing the cruciate dural following cauterization and devascularization. The dura mater is opened from the planum inferiorly based on the SIS. The suprasellar cistern (SC) can be seen and preserved. 

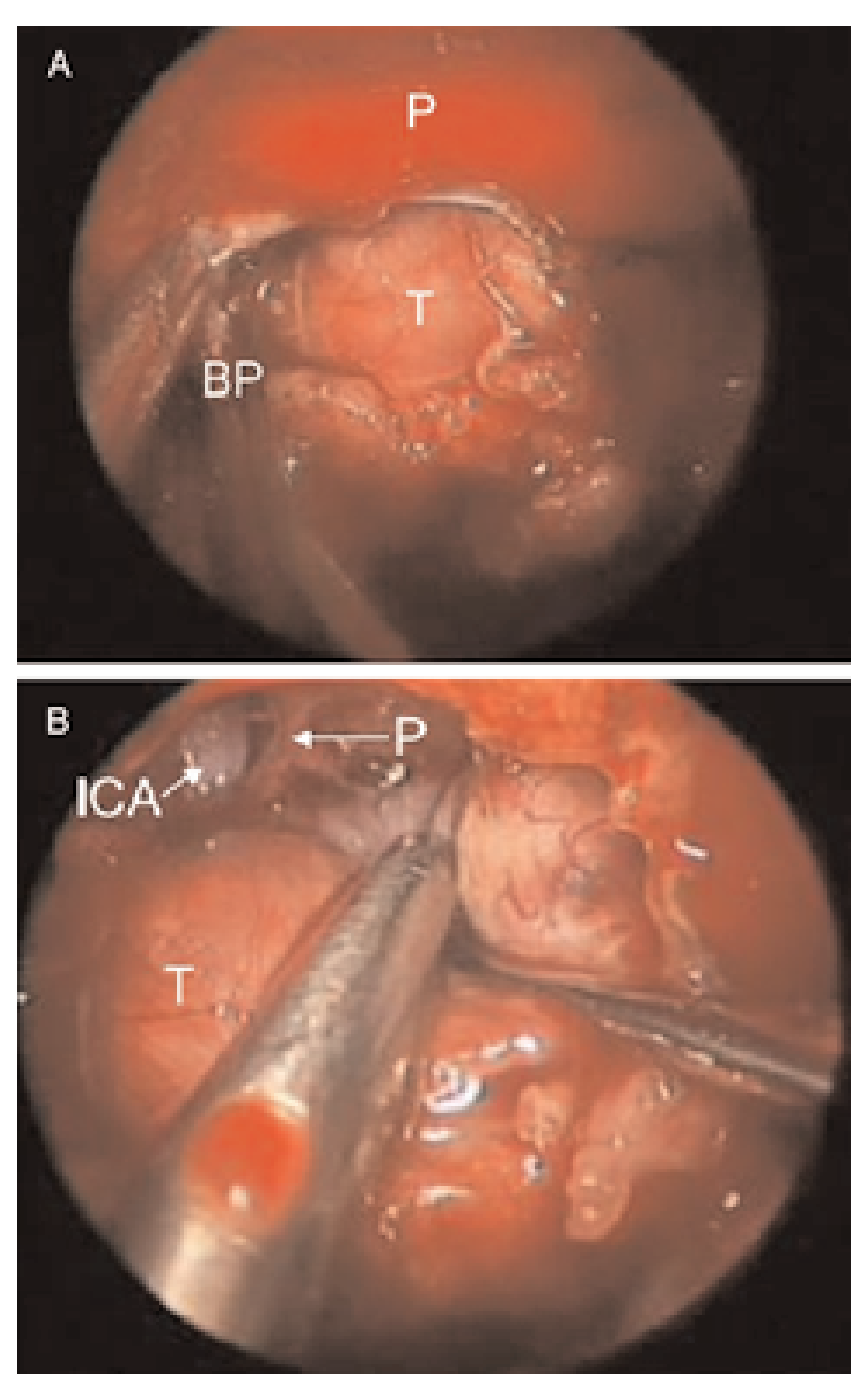

Fig. 8. A: Endoscopic view following opening of the tuberculum and planum $(\mathrm{P})$ dura mater. The tumor $(\mathrm{T})$ can be seen once the suprasellar cistern is opened. B: Endoscopic view demonstrating extracapsular dissection of the tumor to identify the right ICA. Note that a small subchiasmatic perforating vessel $(\mathrm{P})$ can be seen traveling from the ICA over the dome of the tumor to the undersurface of the chiasm. The dural leaf is coagulated using a pistol-grip bipolar (BP) to retract it from the field.

demonstrate the exposure needed and the technique for dissection of vascular structures from the tumor capsule.

\section{Technical Considerations.}

Extradural Approach. This module extends the rostral extension of the previous approach to the level of the crista galli. Following the transplanum module, the transcribriform approach can be initiated by resecting the attachment of the anterior portion of the nasal septum to the skull base. It is likely that olfaction has already been compromised by the disease entity in question. The frontoethmoidal recess is identified and the skull base is drilled from a rostral-to-caudal direction to mitigate the obscuring effects of dripping blood, thus optimizing visualization. The AEA is purposefully identified and sacrificed to provide for tumor devascularization (Fig. 14). If necessary, the back wall of the
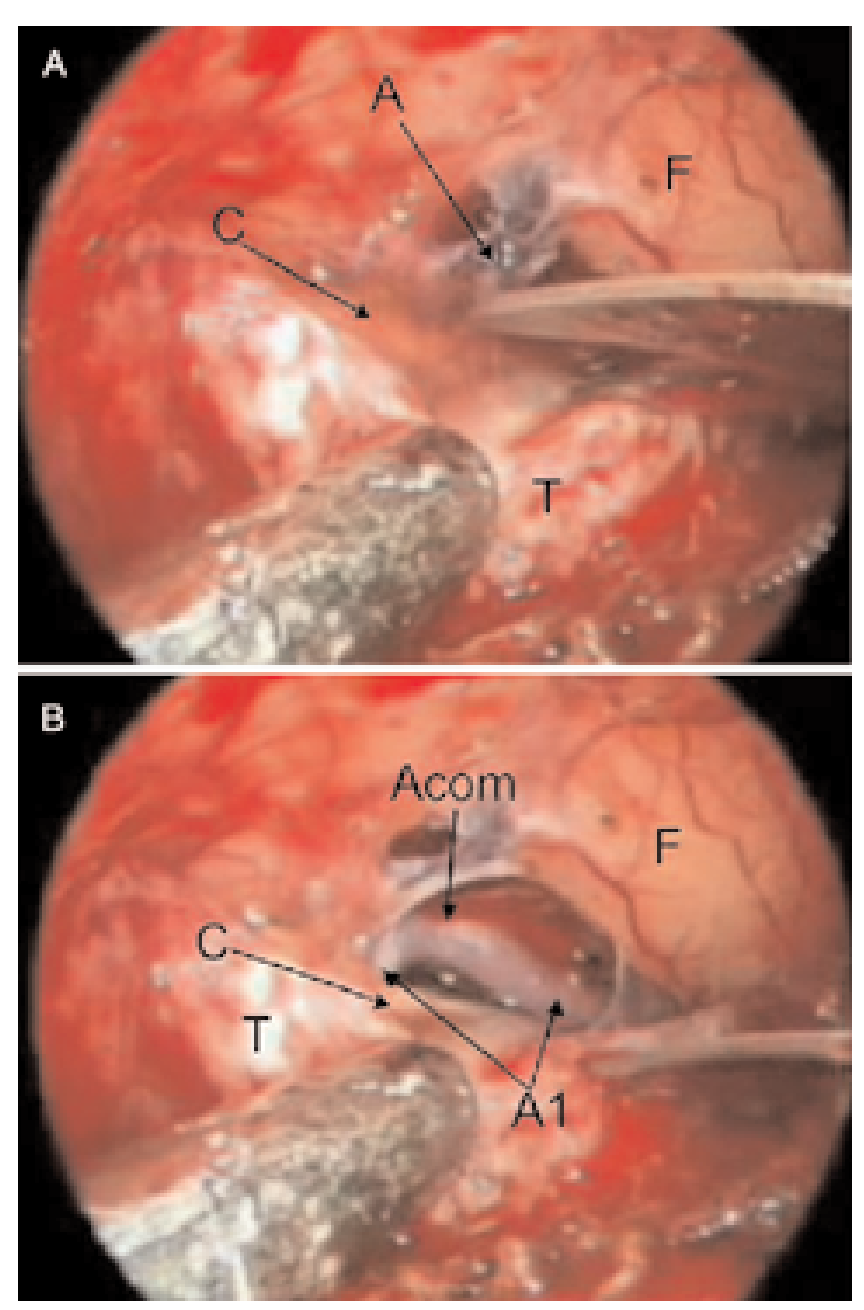

Fig. 9. A: Endoscopic view obtained after initial debulking of the tumor showing the technique for extracapsular sharp dissection performed using scissors to release the arachnoid bands (A) tethering the tumor capsule $(\mathrm{T})$ to the chiasm $(\mathrm{C})$ and frontal lobe $(\mathrm{F})$. $\mathrm{B}$ : Endoscopic view showing the operating field after arachnoidal sharp dissection. Note the position of the ACoA (Acom) and the $\mathrm{A}_{1}$ segments (A1) of the ACAs. The tumor capsule has been sharply dissected from these vessels and the frontal lobe. Arachnoid bands tethering tumor to the chiasm can still be seen.

frontal sinus can be accessed and/or removed via the frontoethmoidal recess.

The olfactory sulcus extends on both sides of the crista galli, from the cribriform plate rostrally to the anterior margin of the planum caudally. To facilitate the drilling of this bone all the overlying soft tissue must be removed. This consists of the small branches of the ethmoidal arteries and the olfactory filaments entering and exiting the crista galli, respectively. Coagulation of these vessels also further aids in devascularization. The cribriform plate is removed bilaterally, leaving the crista galli in the midline, which can extend for a variable depth into the intracranial cavity. The crista galli can be particularly prominent in the case of olfactory groove meningiomas with secondary hyperostosis. Removal of the crista galli will involve additional drilling internally, and then fracture once it has become eggshell thin (Fig. 15). 

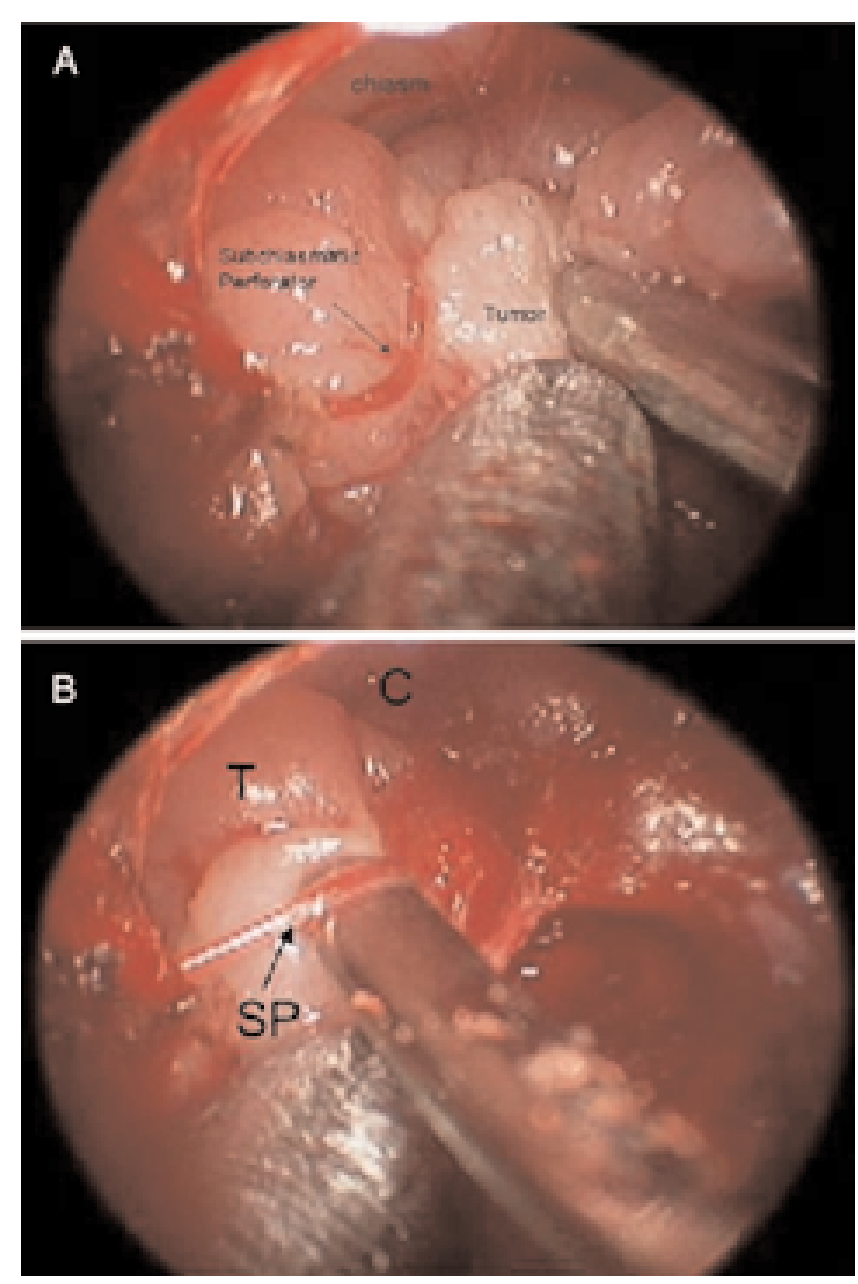

Fig. 10. A: Endoscopic view showing a small subchiasmatic perforating vessel draped over the dome of the tumor. B: Endoscopic view showing that after adequate debulking the tumor capsule $(\mathrm{T})$ can be mobilized to separate this small subchiasmatic perforating vessel (SP) by using endoneurosurgical techniques and to preserve it as it travels to the undersurface of the chiasm (C).

Resection of the crista galli and cribriform plate augmented with the transplanum module (if required) creates a large single cavity along the anterior skull base. The exposed dura mater is thoroughly coagulated, which, in combination with the AEA and PEA ligations, is critical for devascularization. This leaves the anterior falcine artery as the primary residual supply to the tumor, in addition to any cortical vessels parasitized from the anterior circulation.

Intradural Dissection. Following coagulation, the dura mater is opened on both sides of the falx individually. The midline is kept intact intentionally because the tumor in this region is still vascularized. Internal tumor debulking is sequentially performed on each side, exposing the free edge of the falx bilaterally. At this point, the feeding vessels arising from the anterior falcine artery are coagulated along with the falx. The falx is transected to provide a single intradural working cavity. The tumor is debulked starting in the midline. The dura mater anterior to the brain/tumor interface is left intact to prevent herniation of the brain,
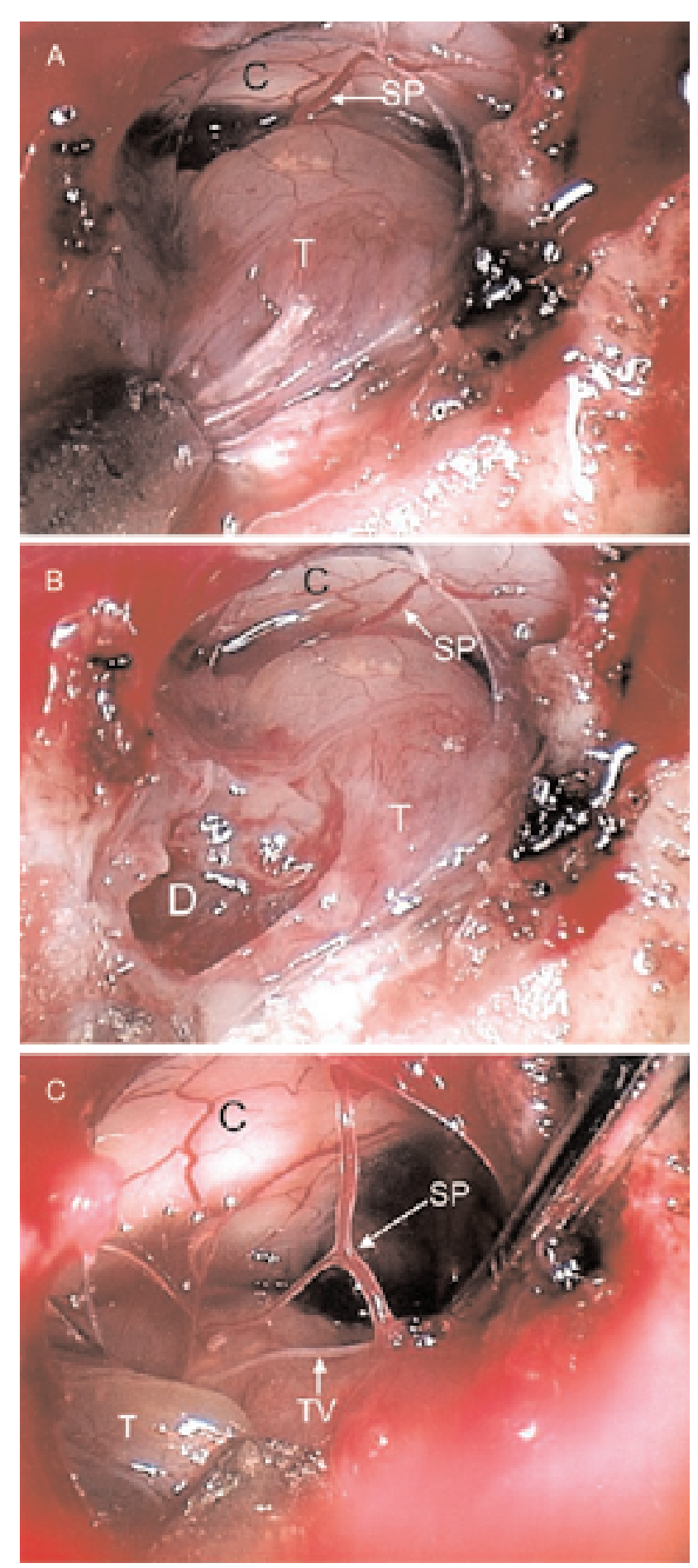

Fig. 11. Endoscopic views showing a small subchiasmatic perforating vessel (SP) traveling to the undersurface of the chiasm (C). A: The initial position of the subchiasmatic perforating vessel draped over the dome of the tumor (T) and adherent to the chiasm is depicted. Note the gentle countertraction being applied to the inferior portion of the tumor capsule to allow for mobilization. B: After initial debulking (D) of the tumor, the subchiasmatic perforating artery is better visualized. C: The initial perforating vessel is entirely separate from the tumor capsule and supplies the chiasm, and a small tumor feeding vessel (TV) supplying the tumor in a deeper plane can be seen. This view shows that this tumor vessel is coagulated and the chiasmatic perforating artery is preserved. 


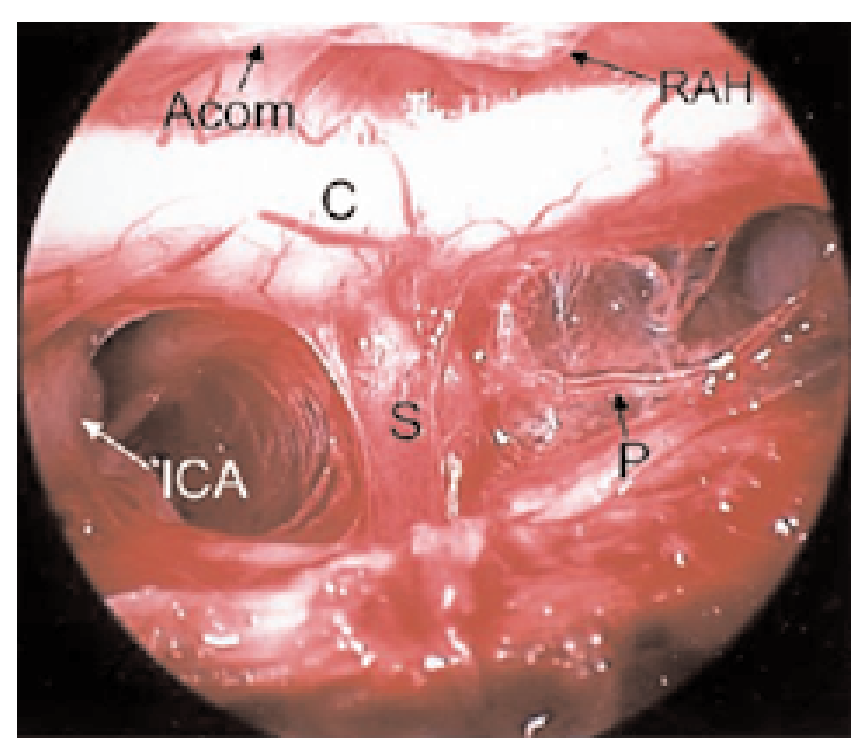

Fig. 12. Endoscopic view obtained after tumor resection. The ACoA (Acom) and the recurrent artery of Huebner (RAH) can be seen above the chiasm (C). A small perforating vessel (P) is preserved and can be seen supplying the infundibulum (S). Also note the preservation of the small perforating arteries on the undersurface of the chiasm. The right ICA can also be seen.

which will obscure visualization. During the extracapsular dissection of large tumors, there is very often subpial invasion of the lesion into the overlying cortex. Therefore, in these cases a subpial dissection must be performed using No. 4 French catheters for suction and fine endoneurosurgical bipolar cauteries under low heat. This dissection can proceed to the level of the interhemispheric fissure along the superior pole of the tumor. Care should be taken because the $\mathrm{A}_{2}$ segments and the frontopolar artery will be draped over the tumor surface (Fig. 16).

It is very useful after an initial adequate internal debulking to proceed toward the parasellar space caudally (inferior pole). This will allow access to the parasellar cistern and identification of key neurovascular landmarks described in the Transtuberculum/Transplanum Approach section. Identification of the optic nerves and ACoA may greatly facilitate the extracapsular dissection of the $\mathrm{A}_{2}$ segments along the interhemispheric fissure from a preferred proximal-to-distal direction. This provides proximal control during the vascular dissection along the capsule interface. The $70^{\circ}$ endoscope is invaluable for the most anterior and rostral dissection of the tumor. When this scope is needed, it is placed at the 6-o'clock position and the suction is moved to 12 o'clock, inverting the previous positions in the patient's right naris (Video 6).

Video 6. Intradural transcribriform dissection. (Click here to view with Windows Media Player and a broadband connection, here to view with a dial-up connection, on here to view with

RealPlayer.)

Staged tumor removal may be an excellent option, especially for exceptionally large tumors in which circumferential extracapsular dissection is not possible because of growth along the sagittal or coronal planes (very "tall and wide tumors"). In these situations, pulsations from all seg-

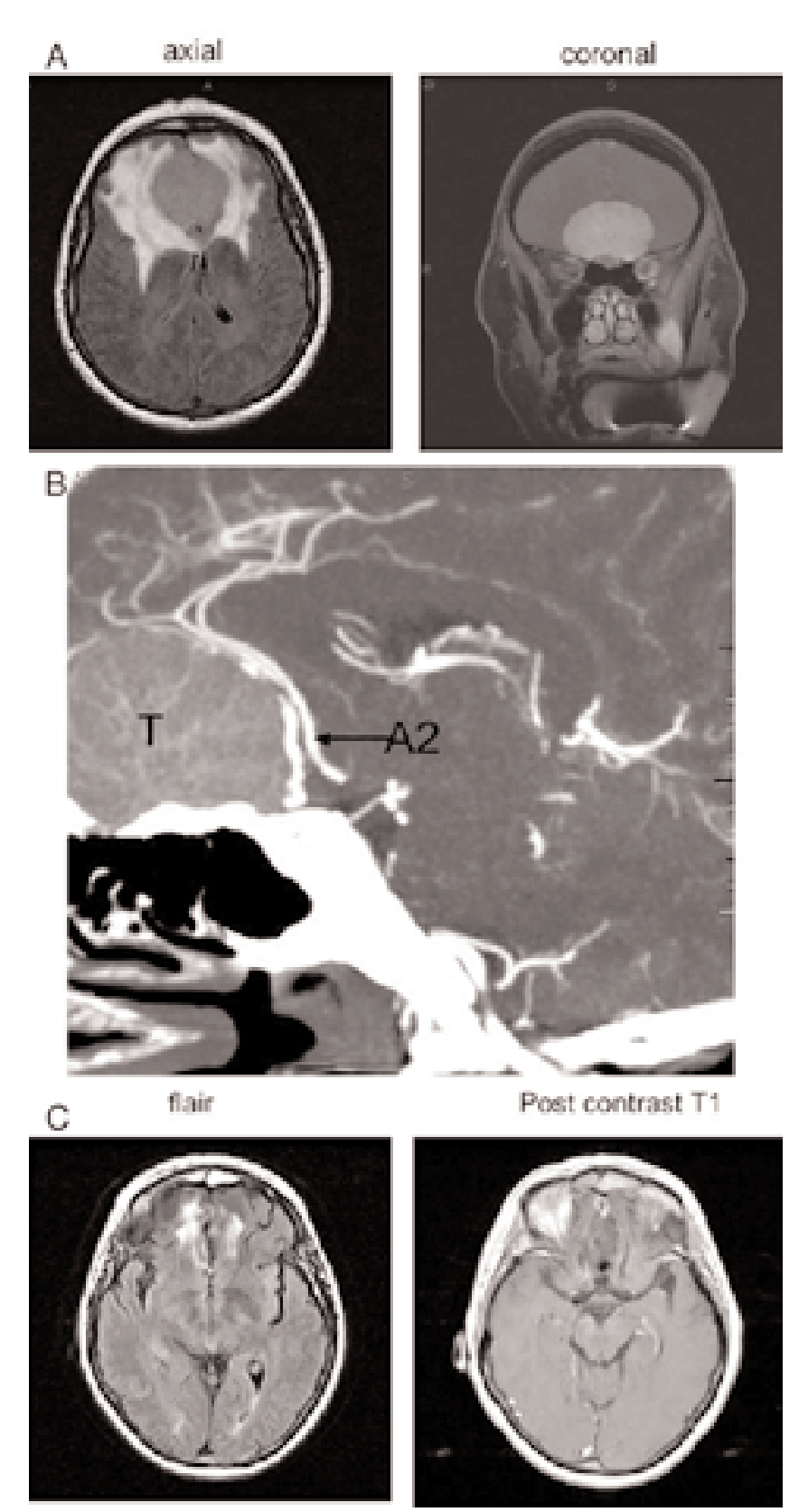

Fig. 13. A: Preoperative MR images demonstrating a large olfactory groove meningioma. Note the significant bifrontal edema as seen on the axial fluid-attenuated inversion-recovery (flair) sequence. On the coronal view note the edema reaching vertically to the level of the corpus callosum and its horizontal extent to the level of the midorbit. Also note the hyperostosis of the anterior base of the tumor on the coronal view. B: Computerized tomography angiography demonstrating the adherence of the second segments of the ACA (A2) on the surface of the tumor (T). C: Postoperative MR images obtained at the 2-year follow-up visit. Note the significant resolution of the bifrontal edema as seen on the axial fluid-attenuated inversion-recovery sequence. The postcontrast $\mathrm{T}_{1^{-}}$ weighted sequence does not reveal evidence of tumor recurrence or residual lesion. 

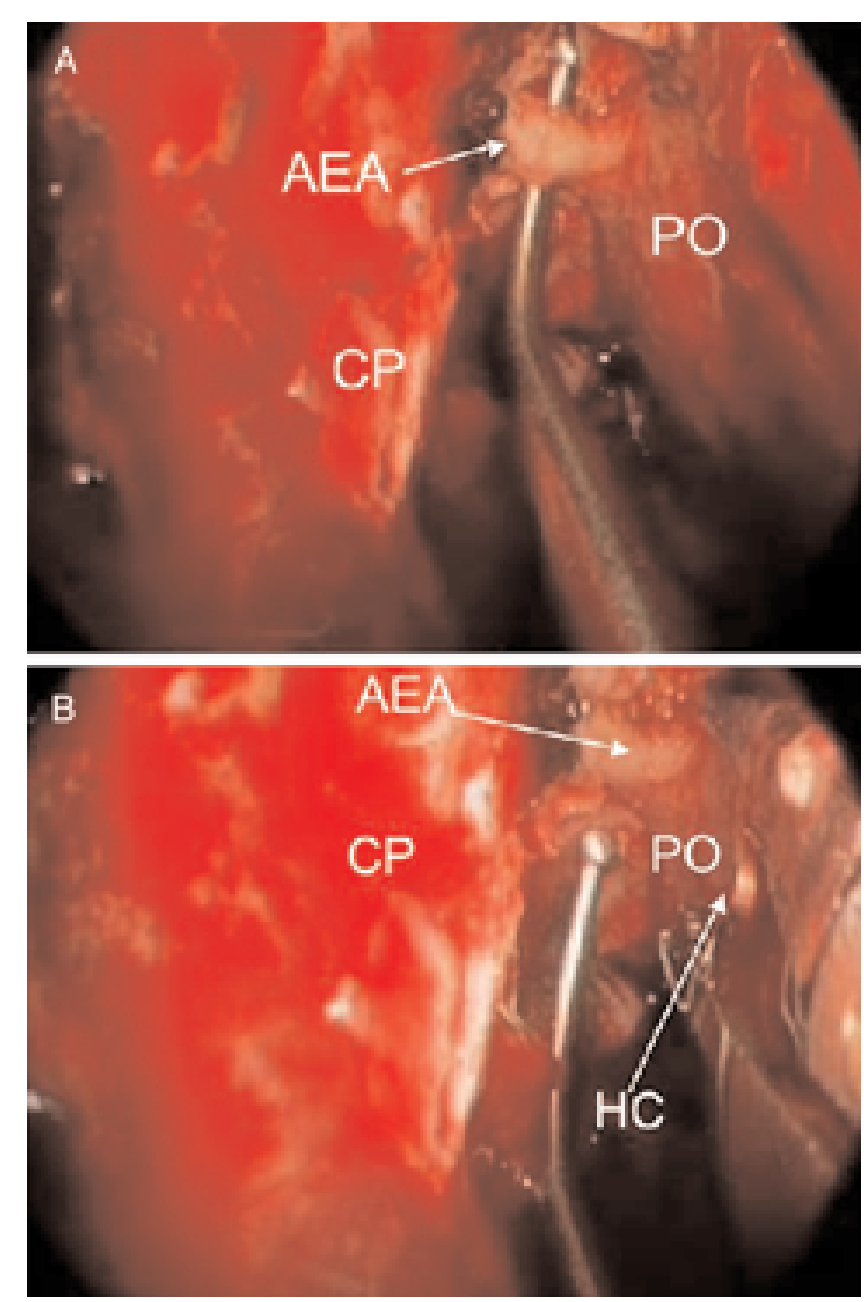

Fig. 14. A: Endoscopic view using a $70^{\circ}$ scope showing the key relationships along the left olfactory sulcus. The cribriform plate $(\mathrm{CP})$ is seen medially and the AEA is also seen. This artery can be seen as it emerges from the orbit after the lamina papyracea has been removed. $\mathrm{B}$ : The periorbita (PO) can be seen laterally and a hemoclip (HC) is applied just at the level at which the AEA emerges from the periorbita.

ments of the tumor cavity following initial debulking should be observed. If this occurs, the tumor will likely migrate in from all directions to fill the cavity created. The first stage is not terminated until such complete pulsations are seen.

\section{DISCUSSION}

In this report we have outlined our 7-year experience in developing a modular approach to the ventral skull base from the sella turcica to the crista galli by using a fully endoscopic and completely transnasal approach. We have demonstrated that the modular approaches are based on specific anatomical corridors and have outlined the key anatomical principles and technical nuances for each region.

Starting with the approach to the pituitary, we have detailed a general binasal exposure of the sphenoid sinus and

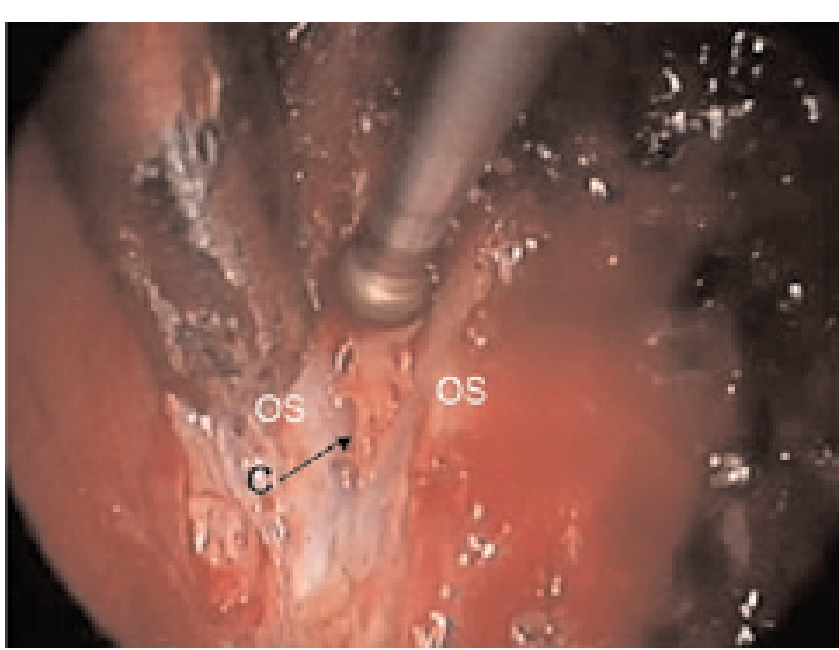

Fig. 15. Endoscopic view using a $70^{\circ}$ scope after clip ligation of the AEAs bilaterally. The cribriform plate over the olfactory sulcus (OS) has been removed bilaterally. The crista galli (C) is drilled from an inferior to superior direction as it penetrates the anterior skull base.

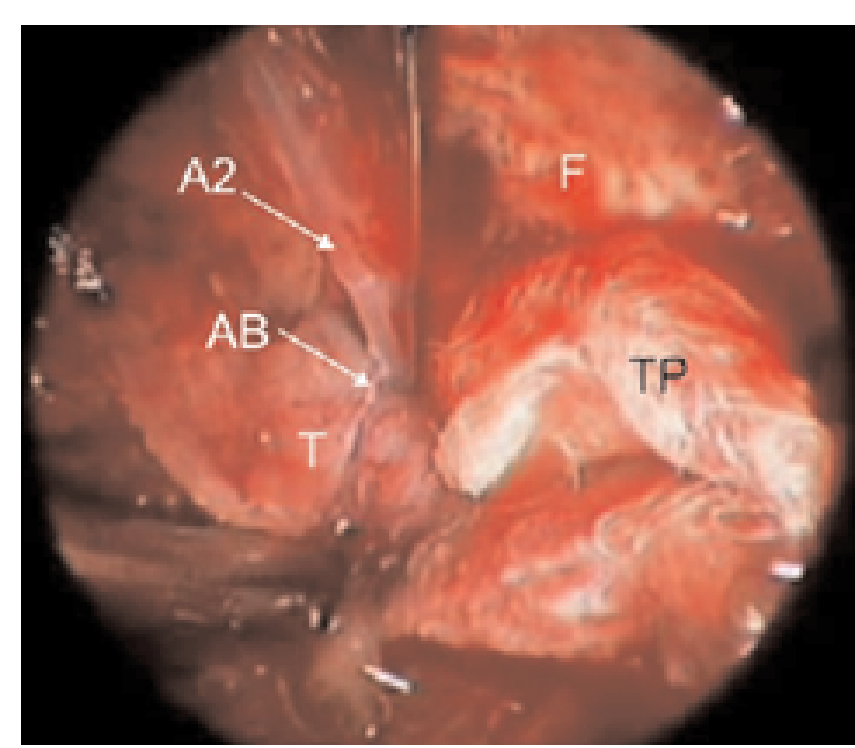

Fig. 16. Endoscopic view using a $70^{\circ}$ scope showing the relationship of the tumor capsule to the second segment of the ACA (A2) as it courses through the interhemispheric fissure (F). Note the small arachnoid bands (AB) attaching the $A_{2}$ to the tumor capsule. Once the tumor has been separated from adjacent structures, a Teflon pledget (TP) is placed to maintain the position. These should be removed at the end of the surgery after tumor resection.

sella turcica. Use of endoneurosurgical techniques necessitates a wide sphenoid opening, which becomes the standard approach for all the expanded modules. Extending anteriorly, we have outlined the feasibility of dealing with intraand extradural lesions of the planum and cribriform regions.

The importance of maintaining strict microneurosurgical techniques in all endoneurosurgical cases has been emphasized. The specific approaches and instrument modifica- 
tions that allow for endoscopic bimanual dissection, internal debulking, capsular mobilization, and extracapsular sharp dissection have been outlined and form the backbone of the expanded endonasal approaches.

\section{CONCLUSIONS}

The goal of this report is to demonstrate the feasibility of the endoscopic approaches to the anterior skull base. We have provided the technical and anatomical nuances and detailed the endoneurosurgical techniques. Also, the important outcome studies are underway to determine the clinical efficacy of this work.

\section{References}

1. Cappabianca P, Alfieri A, Colao A, et al: Endoscopic endonasal transsphenoidal surgery in recurrent and residual pituitary adenomas: technical note. Minim Invasive Neurosurg 43:38-43, 2000

2. Cappabianca P, Cavallo LM, Colao A, et al: Endoscopic endonasal transsphenoidal approach: outcome analysis of 100 consecutive procedures. Minim Invasive Neurosurg 45: 193-200, 2002

3. Carrau RL, Jho HD, Ko Y: Transnasal-transsphenoidal endoscopic surgery of the pituitary gland. Laryngoscope 106: 914-918, 1996

4. Couldwell WT, Weiss MH, Rabb C, et al: Variations on the standard transsphnoidal approach to the sellar region, with emphasis on the extended approaches and parasellar approaches: surgical experience on 105 cases. Neurosurgery 55: 539-550, 2004

5. de Divitiis E, Cappabianca P (eds): Endoscopic Endonasal Transsphenoidal Surgery. New York: Springer-Verlag, 2003
6. Frank G, Pasquini E, Mazzatenta D: Extended transsphenoidal approach. J Neurosurg 95:917-918, 2001

7. Guiot G, Rougerie J, Fourestier M, et al: Explorations endoscopiques intracranienes. Presse Med 71:1225-1228, 1963

8. Halves E, Bushe KA: Transsphenoidal operation on craniopharyngiomas with extrasellar extensions. The advantage of the operating endoscope. Acta Neurochir Suppl 28:362, 1979

9. Hardy J: La chirurgie de l'hypophyse parvoir transsphenoidale ouverte. Etude comparative de deux modalites techniques. Ann Chir 21:1011-1022, 1967

10. Hirsch O: Endonasal method of removal of hypophyseal tumors. With a report of two successful cases. JAMA 55: 772-774, 1910

11. Jho HD: Endoscopic transsphenoidal surgery. J Neurooncology 54:187-195, 2001

12. Jho HD, Carrau RL: Endoscopic endonasal transsphenoidal surgery: Experience with 50 patients. J Neurosurg 87:44-51, 1997

13. Jho HD, Carrau RL, Ko Y, et al: Endoscopic pituitary surgery: an early experience. Surg Neurol 47:213-223, 1996

14. Rosegay H: Cushing's legacy to transsphenoidal surgery. J Neurosurg 54:448-454, 1981

15. Zada G, Kelly DF, Cohen P, et al: Endonasal transsphenoidal approach for pituitary adenomas and other sellar lesions: an assessment of efficacy, safety, and patient impressions. J Neurosurg 98:350-358, 2003

Manuscript received June 8, 2005.

Accepted in final form June 28, 2005.

Address reprint requests to: Amin B. Kassam, M.D., University of Pittsburgh School of Medicine, 203 Lothrop Street, Suite 500, Pittsburgh, Pennsylvania 15213. email: kassamab@upmc.edu. 